\title{
Vertical propagation of baroclinic Kelvin waves along the west coast of India
}

\author{
D Nether ${ }^{1,2, *}$ and D ShankAR ${ }^{1}$ \\ ${ }^{1}$ National Institute of Oceanography, Goa 403 004, India. \\ ${ }^{2}$ Ecole Normale Supérieure, Paris, France. \\ *e-mail: daniel.nethery@ens.fr
}

\begin{abstract}
A linear, continuously stratified ocean model is used to investigate vertical propagation of remotely forced, baroclinic Kelvin waves along the Indian west coast. The extent of vertical propagation over the length of the coast is found to be an increasing function of the forcing frequency. Simulations show that, over the length of the Indian west coast, vertical propagation is limited at annual and semi-annual periods, but significant at periods shorter than about 120 days. This has two major consequences. First, the depth of subsurface currents associated with these frequencies varies substantially along the coast. Second, baroclinic Kelvin waves generated in the Bay of Bengal at periods shorter than about 120 days have negligible influence on surface currents along the north Indian west coast.
\end{abstract}

\section{Introduction}

Current measurements to the south of Sri Lanka reveal significant annual and semi-annual components. Schott et al (1994) and Reppin et al (1999) found that these components together account for around $50 \%$ of observed current variability, with near-surface values reaching as high as $80 \%$. Such pronounced annual and semi-annual variability is a characteristic of north Indian Ocean circulation.

One manifestation of this variability is the annual cycle of sea surface height in the vicinity of the Lakshadweep Islands (off southwest India). Using a linear model on the equatorial beta-plane, Shankar and Shetye (1997) showed that the general features of the Lakshadweep high and low, including its formation and subsequent westward propagation, result from the annual and semi-annual variability of remotely forced currents south of Sri Lanka and the 'leaking' of Rossby waves from the coastal wave guide.

The current measurements reported by Schott et al (1994) also show visibly high variance at shorter than semi-annual periods. Shankar and Shetye (1997) found that while variability at shorter than semi-annual periods may contribute to the formation of the Lakshadweep high and low, significant energy remains trapped to the Indian west coast. Model simulations (McCreary et al 1993; Shankar and Shetye 1997; Shankar et al 2002) have long suggested the importance of remote forcing from the Bay of Bengal for the seasonal cycle of the West India Coastal Current. Recent analysis of tide-gauge data along the Indian west coast also indicates that the influence on sea level of remote forcing is at least as important as that of local wind forcing, even at periods as short as 10 days (S R Shetye, personal communication 2006).

Given the influence of remote forcing, it is important to consider how coastal Kelvin waves might affect the vertical structure of currents along the Indian west coast. Romea and Allen (1983) demonstrated that baroclinic coastal Kelvin waves in a continuously stratified ocean propagate vertically. This paper studies such vertical propagation in the specific context of the Indian west coast using a

Keywords. Arabian Sea; Indian Ocean; west India coastal current; monsoon current; equatorial oceanography; remote forcing; modelling; monsoons; oceanography. 

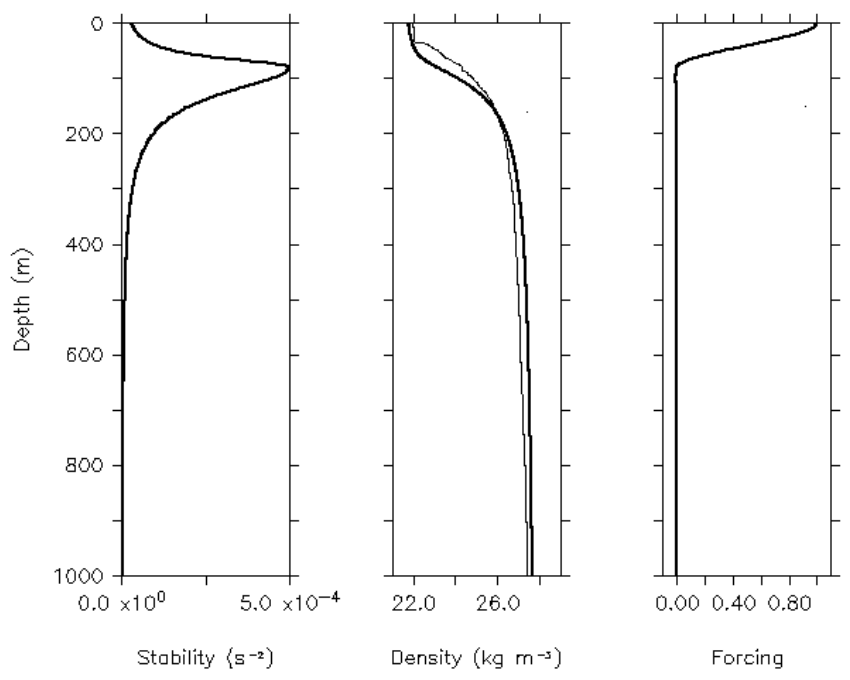

Forcing

Figure 1. The stability or buoyancy frequency squared $N_{b}^{2}$ profile (left), the background density $\rho_{b}-1000$ profile (thick curve, centre) and the forcing vertical profile $\Psi$ (right) used in all simulations. A typical $\sigma_{t}$ profile reported by Shenoi et al (2005) is also shown (thin curve, centre).

linear, continuously stratified model on the equatorial beta-plane.

\section{The model ocean}

The model is the linear, continuously stratified system of McCreary (1981) as adapted by Shankar and Shetye (1997) to study circulation along the Indian west coast. A flat-bottom ocean of depth $D=3000 \mathrm{~m}$ is situated on the equatorial $\beta$-plane in the Northern Hemisphere. Cartesian coordinates are used with $x$ positive eastward, $y$ positive northward and $z$ positive vertically upward.

The hydrostatic approximation is assumed and the equations of motion are linearised about a background stable density profile $\rho_{b}(z)$ with associated buoyancy or Brunt-Väisälä frequency $N_{b}(z)$ (figure 1, left and centre). The background density profile is chosen to resemble profiles observed off the Indian west coast (Shenoi et al 2005). The profile of $N_{b}^{2}$ attains a maximum at 80-m depth, which we refer to as the thermocline depth $D_{t}$.

Motion is considered to be outside frictional boundary layers, allowing horizontal viscosity to be neglected, while the coefficients of vertical viscosity and diffusion are taken to be inversely proportional to the buoyancy frequency squared, so that

$$
\nu=\kappa=\frac{M_{v}}{N_{b}^{2}},
$$

where $M_{v}=1.3 \times 10^{-4} \mathrm{~cm}^{2} \mathrm{~s}^{-3}$, the value adopted by Shankar et al (1996) and Shankar and Shetye
(1997). The sensitivity of solutions to $M_{v}$ is discussed in section 4.4.

These mathematically convenient definitions of vertical mixing allow solutions to be found as expansions of vertical modes $\psi_{n}(z)$ of the form

$$
\begin{aligned}
& p=\sum_{n=0}^{\infty} p_{n}(x, y, t) \psi_{n}(z), \\
& u=\sum_{n=0}^{\infty} u_{n}(x, y, t) \psi_{n}(z), \\
& v=\sum_{n=0}^{\infty} v_{n}(x, y, t) \psi_{n}(z), \\
& w=\sum_{n=0}^{\infty} w_{n}(x, y, t) \int_{-D}^{z} \psi_{n}(\xi) d \xi \\
& \rho=\sum_{n=0}^{\infty} \rho_{n}(x, y, t) \frac{d \psi_{n}}{d z}(z) .
\end{aligned}
$$

Here, $u, v$ and $w$ are zonal, meridional and vertical velocities, and a factor of $\rho_{0}$, a typical value of $\rho_{b}$, has been absorbed into the definitions of pressure and density, so that $p$ has units of (potential) energy per unit mass and $\rho$ represents (dimensionless) density anomaly.

The vertical modes $\psi_{n}$ are the eigenfunctions of the equation

$$
\left(\frac{\left(\psi_{n}\right)_{z}}{N_{b}^{2}}\right)_{z}=-\frac{1}{c_{n}^{2}} \psi_{n}
$$

subject to the boundary conditions $\left(\psi_{n}\right)_{z}=0$ at $z=-D$ and $z=0$, where subscripts $z$ denote partial differentiation and $c_{n}$ is the Kelvin wave, or characteristic, speed of the $n$th mode.

Equations for the coefficients of the $n$th mode are

$$
\begin{aligned}
& i \omega_{n} u_{n}-f v_{n}+p_{n x}=\tau_{n}^{(x)}, \\
& i \omega_{n} v_{n}+f u_{n}+p_{n y}=\tau_{n}^{(y)}, \\
& i \omega_{n} \frac{p_{n}}{c_{n}^{2}}+u_{n x}+v_{n y}=0,
\end{aligned}
$$

where subscripts $x$ and $y$ denote partial differentiation, $f=\beta y$ is the Coriolis parameter, $\tau_{n}^{(x / y)}$ is the zonal/meridional wind stress coupled to the $n$th 
mode and $\omega_{n}$ is as defined in (9) below. Equations for the remaining coefficients,

$$
\begin{aligned}
& w_{n}=i \omega_{n} \frac{p_{n}}{c_{n}^{2}}, \\
& \rho_{n}=\frac{p_{n}}{g},
\end{aligned}
$$

where $g$ is the acceleration due to gravity, are determined once the pressure field is known.

See McCreary (1981) for a complete discussion of the model and assumptions.

This paper is concerned with baroclinic, remotely forced coastal Kelvin waves along the Indian west coast. As such, we neglect the $n=0$ term in the sums (2) and set the local wind forcing $\tau_{n}^{(x / y)}=0$ for all $n$ in (4). Following Shankar and Shetye (1997), we take the Indian west coast to be a meridional eastern boundary, situated along $x=0$, which terminates north of the Equator. We also model the monsoon current south of Sri Lanka as a purely zonal flow with a Gaussian meridional profile that varies sinusoidally in time. Specifically, we apply the boundary condition

$$
\begin{aligned}
u(x & =0, \eta, z, t) \\
& =u_{0} \exp \left[-\frac{\left(\eta-\eta_{0}\right)^{2}}{2 \gamma}\right] \Psi(z) e^{i \sigma t}, \quad 0<\gamma<\frac{1}{2},
\end{aligned}
$$

where

$$
\eta=\frac{y}{\delta_{n}}=y \sqrt{\frac{\beta}{c_{n}}}
$$

and $\delta_{n}$ is the $n$ th-mode $\beta$-plane Rossby radius of deformation.

This condition represents a zonal flow into or out of the domain $(x \leq 0)$. The profile is symmetric about a forcing latitude at distance $y_{0}$ from the Equator. Following Shankar and Shetye (1997), we take the forcing latitude to be $5^{\circ} \mathrm{N}$ and set $\gamma=2.5 \times 10^{-2}$.

The choice of $\gamma$ ensures that the zonal flow is confined to a narrow region around the forcing latitude that corresponds to the zone of the monsoon current south of Sri Lanka (figure 2). Sufficiently far from the forcing latitude, the zonal flow along $x=0$ is negligible. To the north, this is assumed to represent the absence of normal flow along the eastern boundary, or Indian west coast. To the south, the choices of forcing latitude and $\gamma$ ensure that the zonal forcing lies within the monsoon-current regime south of Sri Lanka and is negligible in the equatorial zone.
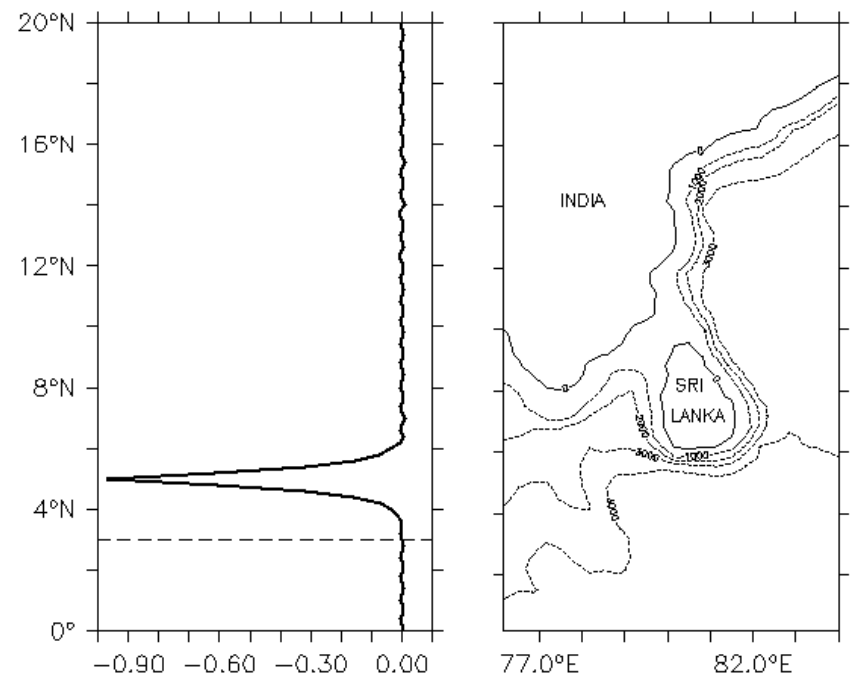

Figure 2. Boundary condition (7) (left) and the geographic context in which it is applied (right). The boundary condition specifies the zonal velocity $u$ along $x=0$, represented here at time $t=T / 2$, where $T$ is the period of variability, and normalised with respect to the forcing amplitude $u_{0}$. The dashed line indicates the limit of the equatorial zone. The boundary condition has been calculated with $N=50$ vertical modes and $M=12,500$ meridional modes.

The extent of the equatorial zone is conveniently taken to be the first-mode $\beta$-plane Rossby radius of deformation $\delta_{1}$, defined in (8) above. For the stability profile used in simulations, $\delta_{1} \approx 3^{\circ}$ (figure 2 , left, dashed line). The location of the southern tip of Sri Lanka, at around $6^{\circ} \mathrm{N}$, is therefore sufficiently far north to allow coastal Kelvin waves, generated in the Bay of Bengal, to propagate around Sri Lanka and into the Arabian Sea without interacting with the equatorial zone (Shankar et al 2002). (If the southern tip of Sri Lanka was situated at $4^{\circ} \mathrm{N}$ instead of $6^{\circ} \mathrm{N}$, the monsoon-current regime would overlap the equatorial zone, resulting in a north Indian Ocean circulation very different from that which we observe.) The negligible zonal flow in the equatorial zone, specified by the boundary condition, enables us to isolate the response of the West India Coastal Current to the monsooncurrent forcing. As we shall see, north of the forcing latitude the response consists of a prominent circulation along the Indian west coast, a consequence of the property that Kelvin waves propagate poleward along an eastern boundary.

Schott et al (1994) reported that currents off the southern tip of Sri Lanka are strongly surfacetrapped. An idealised vertical profile $\Psi(z)$ is defined accordingly, with zero current at the thermocline depth $D_{t}=80 \mathrm{~m}$ and an undercurrent attaining a maximum of $1 \%$ of the surface speed at $100-m$ depth (figure 1). The advantage of this profile is that it allows the extent of vertical propagation at typical forcing periods to be readily assessed. 
With the choice of sinusoidal time-dependent forcing at the boundary, it follows that

$$
i \omega_{n}=i \sigma+\frac{M_{v}}{c_{n}^{2}},
$$

where $M_{v}$ is the vertical mixing parameter defined in (1).

Expanding the boundary condition (7) into vertical modes, yields

$$
\begin{aligned}
u_{n}= & u_{0} \varphi_{n} \sum_{m=1}^{\infty} B_{m}\left[K_{m} \phi_{m+1}(\eta)-\phi_{m-1}(\eta)\right] \\
& \times e^{i\left(k_{m} x+\sigma t\right)} \\
v_{n}= & u_{0} \varphi_{n} \sum_{m=1}^{\infty} B_{m}\left[L_{m} \phi_{m}(\eta)\right] e^{i\left(k_{m} x+\sigma t\right)}, \\
\frac{p_{n}}{c_{n}}= & u_{0} \varphi_{n} \sum_{m=1}^{\infty} B_{m}\left[K_{m} \phi_{m+1}(\eta)+\phi_{m-1}(\eta)\right] \\
& \times e^{i\left(k_{m} x+\sigma t\right)} .
\end{aligned}
$$

Here

$$
\varphi_{n}=\frac{\int_{-D}^{0} \Psi \psi_{n} d z}{\int_{-D}^{0} \psi_{n}^{2} d z}
$$

measures the response of the $n$th mode to forcing, $\phi_{m}$ is the $m$ th normalised Hermite function, the zonal wave number

$$
k_{m}=\frac{\beta}{2 \omega_{n}} \pm \sqrt{\frac{\beta^{2}}{4 \omega_{n}^{2}}+\frac{\omega_{n}^{2}}{c_{n}^{2}}-\frac{2 m+1}{\delta_{n}^{2}}},
$$

with the sign chosen so that the imaginary part of $k_{m}$ is negative, thus ensuring that the boundary waves decay to the west, and

$$
\begin{gathered}
K_{m}=\frac{c_{n} k_{m}-\omega_{n}}{c_{n} k_{m}+\omega_{n}} \sqrt{\frac{m+1}{m}}, \\
L_{m}=i \delta_{n}\left(k_{m}-\frac{\omega_{n}}{c_{n}}\right) \sqrt{\frac{2}{m}} .
\end{gathered}
$$

The coefficients $B_{m}$ depend on the boundary condition at $x=0$. When this condition is as in (7), they satisfy the recursion relation

$$
\begin{aligned}
B_{m}= & B_{m-2} K_{m-2}-\exp \left[\frac{\gamma \eta_{0}^{2}}{2\left(1-\gamma^{2}\right)}\right] \\
& \times \sqrt{\frac{2 \pi \gamma}{1+\gamma}}\left(\frac{1-\gamma}{1+\gamma}\right)^{\frac{m}{2}} \phi_{m}\left[\frac{\eta_{0}}{\sqrt{1-\gamma^{2}}}\right],
\end{aligned}
$$

with $B_{-1}=B_{0}=0$. This relation is obtained by integrating from $\eta_{n}=-\infty$ to $+\infty$, and it completes the solution.

Solutions correspond to the real part of the variables. We present solutions from $5^{\circ} \mathrm{N}$ to $20^{\circ} \mathrm{N}$, taken to represent the length of the Indian west coast. Summations (2) and (10) must be truncated at finite values, denoted by $N$ and $M$. The coefficients $\varphi_{n}$, which eventually always decrease with $n$, may be used to determine an appropriate $N$, while a suitable value of $M$ can be determined by comparing the solution along $x=0$ with the boundary condition (7). For our choice of $\Psi$, we found that $N=50$ and $M=12,500$ are sufficient to ensure convergence.

\section{Results}

In this section, we demonstrate how coastal Kelvin waves of different frequencies would be expected to influence the vertical structure of currents along the Indian west coast. Schott et al (1994) found that current measurements south of Sri Lanka reveal strong annual and semi-annual components. The data also exhibit high variance at periods of 30-40 days, 50-70 days and 120 days. We focus in particular on 30- and 360-day simulations in order to emphasise that the effects of vertical propagation over the length of the Indian west coast become more pronounced with increasing frequency.

Schott et al (1994) reported the amplitudes of the annual and semi-annual components in both current meter measurements and ship-drift data off the southern tip of Sri Lanka. In both cases, the amplitude of each component in the ship-drift data is twice that found in the current meter data at 75-m depth. Specifically, the amplitude of the semi/annual signal is $10 / 50 \mathrm{~cm} \mathrm{~s}^{-1}$ in the shipdrift data and $5 / 25 \mathrm{~cm} \mathrm{~s}^{-1}$ at $75-\mathrm{m}$ depth. The ship-drift record yields the most appropriate values of the surface forcing amplitudes $u_{0}$. We therefore assume that a 2:1 ratio between the ship-drift and current meter data holds at all frequencies. This enables us to use the $75-\mathrm{m}$ depth data to set the values of $u_{0}$ for all simulation periods (see table 1). 
Table 1. Surface forcing amplitudes $u_{0}$ used in simulations.

\begin{tabular}{cc}
\hline $\begin{array}{c}\text { Period } \\
\text { (days) }\end{array}$ & $\begin{array}{c}\text { Surface forcing } \\
\text { amplitude } \mathrm{cms}^{-1}\end{array}$ \\
\hline 30 & 10 \\
60 & 15 \\
120 & 50 \\
180 & 10 \\
360 & 50 \\
\hline
\end{tabular}

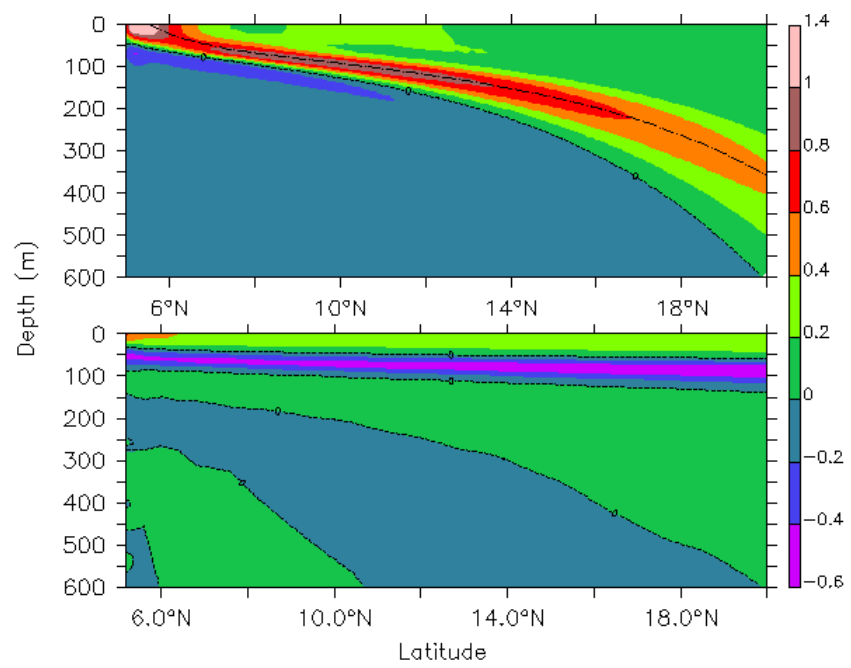

Figure 3. Alongshore velocity fields along the coast $x=0$ at time $t=T / 2$ for periods $T=30$ (top) and $T=360$ (bottom) days, normalised with respect to the forcing surface amplitude $u_{0}$. The beam closely follows the trajectory predicted by (17), shown here for the 30-day period (top, dashed line).

\subsection{Vertical propagation}

The most striking feature of the solutions is the coherent vertical propagation of energy into the deep ocean from the source region centred at the surface at $5^{\circ} \mathrm{N}$. The extent of vertical propagation clearly depends on the forcing period. Figure 3 shows vertical sections of alongshore velocity taken at the coast $x=0$ at time $t=T / 2$ for periods $T=30$ and 360 days. The alongshore velocity is normalised with respect to the forcing surface amplitude $u_{0}$. At this time, the driving zonal current is maximum and directed westward, which, for the annual cycle, corresponds to the situation during the northeast monsoon.

McCreary (1984) demonstrated that, on an $f$ plane, Kelvin-wave 'beams' propagate at slope

$$
\frac{d z}{d y}= \pm \frac{\sigma}{N_{b}(z)}
$$

It follows that a beam bending into the deep ocean follows a pathway given by

$$
z(y)=z\left(y_{0}\right)-\int_{y_{0}}^{y} \frac{\sigma}{N_{b}[z(\xi)]} d \xi
$$

until it reaches the ocean floor. This equation is verified by the coastal Kelvin-wave beams in the solutions, as is readily checked with ray tracing techniques (see figure 3, top, dashed line).

A measure of the vertical propagation in the solutions pertinent to the specific context of the Indian west coast is given by

$$
z\left(5^{\circ} \mathrm{N}\right)-z\left(20^{\circ} \mathrm{N}\right)=\int_{5^{\circ} \mathrm{N}}^{20^{\circ} \mathrm{N}} \frac{\sigma}{N_{b}[z(y)]} d y
$$

for the range of simulation frequencies. The extent of vertical propagation is found to be an increasing function of the forcing frequency (see table 2). Note that at the annual period the extent of vertical propagation is only around 40 meters. This suggests that, at this frequency, the effects of vertical propagation, such as the variation in the upand downwelling cycle over the length of the coast, might be insignificant. This point is taken up in greater detail in the following sections.

The alongshore velocity sections also reveal two interesting aspects of solutions. First, normalised alongshore velocities generally weaken with increasing forcing period $T$. This is due to the leaking of Rossby waves from the coastal waveguide, a process which is dependent on the critical latitude, an increasing function of $T$ (Shankar and Shetye 1997). Second, like their equatorial counterparts, coastal Kelvin-wave beams exhibit a $\pi$ phase shift across the beam with phase increasing towards the surface, as discussed by McCreary (1984).

\subsection{Subsurface currents}

One distinguishing feature of the 360-day cycle alongshore velocity section is the presence of a strong equatorward undercurrent at time $t=T / 2$ (figure 3, bottom). This current is generated

Table 2. Vertical propagation over the length of the Indian west coast.

\begin{tabular}{cc}
\hline $\begin{array}{c}\text { Period } \\
\text { (days) }\end{array}$ & $\begin{array}{c}\text { Vertical } \\
\text { propagation }(\mathrm{m})\end{array}$ \\
\hline 30 & 350 \\
60 & 125 \\
90 & 100 \\
120 & 80 \\
180 & 60 \\
360 & 40 \\
\hline
\end{tabular}


despite the negligible undercurrent in the forcing vertical profile $\Psi(z)$. Moreover, speeds at the core of the undercurrent are greater than those at the surface.

The time series of alongshore velocity at $10^{\circ} \mathrm{N}$ and $20^{\circ} \mathrm{N}$, taken at the coast $(x=0)$, confirms that maximum speeds over the annual cycle do indeed occur in the core of the undercurrent and increase by around $5 \mathrm{~cm} \mathrm{~s}^{-1}$ from $10^{\circ} \mathrm{N}$ to $20^{\circ} \mathrm{N}$ (figure 4 ). The depth of the undercurrent core also increases by around 20 meters over the same distance. It is noteworthy that maximum speeds at the surface and in the undercurrent occur more or less simultaneously along the length of the coast.

The corresponding time series confirms that the situation is quite different for the 30-day cycle (figure 5). While the depth of the subsurface maximum increases by around $200 \mathrm{~m}$ over the distance between $10^{\circ} \mathrm{N}$ and $20^{\circ} \mathrm{N}$, subsurface velocities weaken considerably. Moreover, maximum speeds at and below the surface no longer occur simultaneously. As a result, strong currents at depth flow in the same direction as surface currents over about half of the cycle.

The 120-day cycle (not shown) represents an intermediate case. At $10^{\circ} \mathrm{N}$ surface and subsurface currents peak more or less simultaneously, with the flow at and below the surface in opposite directions. However, this is no longer the case at $20^{\circ} \mathrm{N}$. Here subsurface flow is maximum when the surface current is zero. As for the 360-day cycle, maximum current speeds also increase to the north.

Note that all time series exhibit upward phase propagation, consistent with the downward propagation of energy.

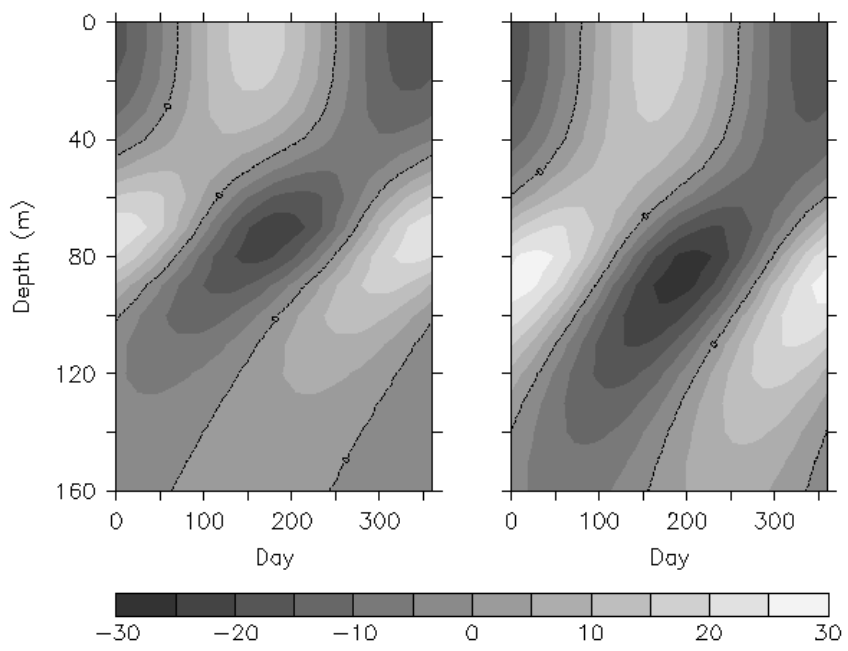

Figure 4. Time series of alongshore velocity $\left(\mathrm{cm} \mathrm{s}^{-1}\right)$ at $10^{\circ} \mathrm{N}$ (left) and $20^{\circ} \mathrm{N}$ (right) for period $T=360$ days and forcing surface amplitude $u_{0}=50 \mathrm{~cm} \mathrm{~s}^{-1}$. The subsurface current maximum increases to the north and an undercurrent is present throughout the cycle.
How subsurface currents vary along the coast therefore depends on the forcing period. At periods of 90 days or more, the subsurface current maximum attained over the cycle increases to the north. This may be explained by considering the path of the beam, which, as discussed in section 3.1 , is confined to the upper ocean. As the beam enters the depth range of the thermocline, the slope of the beam, given by (16), decreases, restricting the vertical propagation of energy and causing the amplitude of the alongshore velocity cycle to increase. This decrease in vertical propagation, combined with the increased coastal trapping of energy as the radius of deformation $\delta_{n}$ decreases away from the Equator, is responsible for the poleward increase in subsurface currents at these periods.

\subsection{Vertical velocity cycle}

One of the most important aspects of baroclinic Kelvin waves is their influence on up- and downwelling in coastal regions. The variation in the vertical velocity cycle over the length of the coast is therefore an important criterion by which to judge the significance of vertical propagation at a particular period.

The time series of the 30-day vertical velocity cycle at $10^{\circ} \mathrm{N}$ and $20^{\circ} \mathrm{N}$ show that the amplitude of the cycle, as well as the depth at which the maximum speed occurs, increase substantially to the north (figure 6 ). At $10^{\circ} \mathrm{N}$ the maximum vertical speed of $1.2 \mathrm{~m} \mathrm{day}^{-1}$ occurs at $110-\mathrm{m}$ depth, while at $20^{\circ} \mathrm{N}$ the maximum speed of $2.6 \mathrm{~m} \mathrm{day}^{-1}$ occurs at 500-m depth.
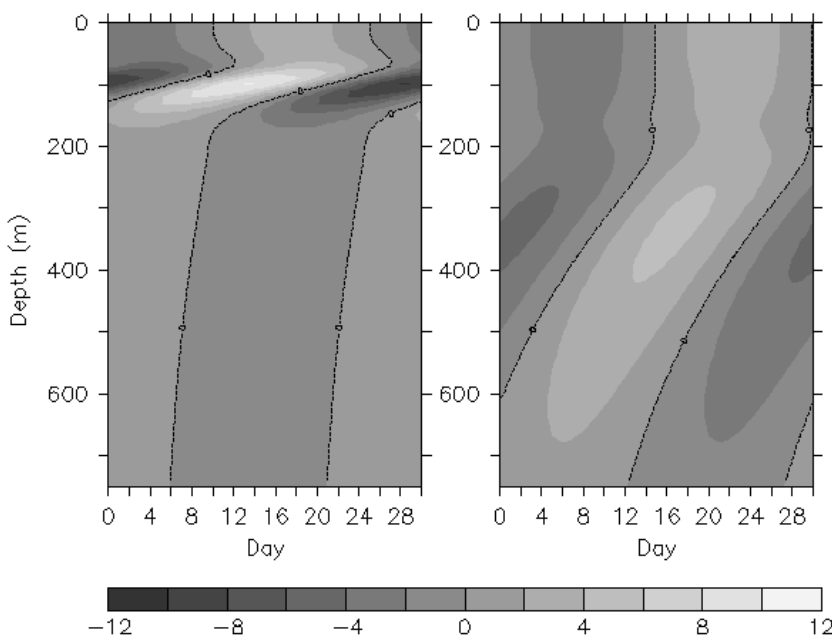

Figure 5. Time series of alongshore velocity $\left(\mathrm{cm} \mathrm{s}^{-1}\right)$ at $10^{\circ} \mathrm{N}$ (left) and $20^{\circ} \mathrm{N}$ (right) for period $T=30$ days and forcing surface amplitude $u_{0}=10 \mathrm{~cm} \mathrm{~s}^{-1}$. The depth of the subsurface current maximum increases significantly to the north. The core of the subsurface current is in the same direction as the surface current over about half of the cycle. 

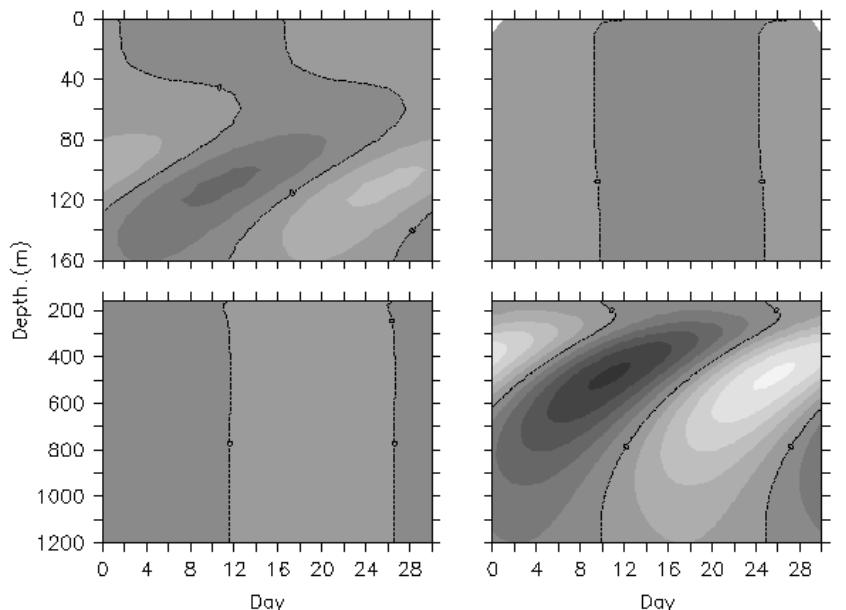

Day

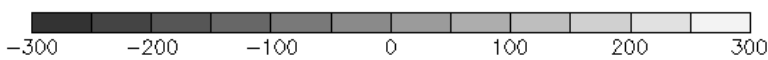

Figure 6. The 30 -day vertical velocity cycle at $10^{\circ} \mathrm{N}$ (left, $\mathrm{cm} \mathrm{day}^{-1}$ ) and at $20^{\circ} \mathrm{N}$ (right, $\mathrm{cm} \mathrm{day}{ }^{-1}$ ) along the coast. The amplitude of the cycle and the depth at which the maximum speed occurs increase significantly to the north.
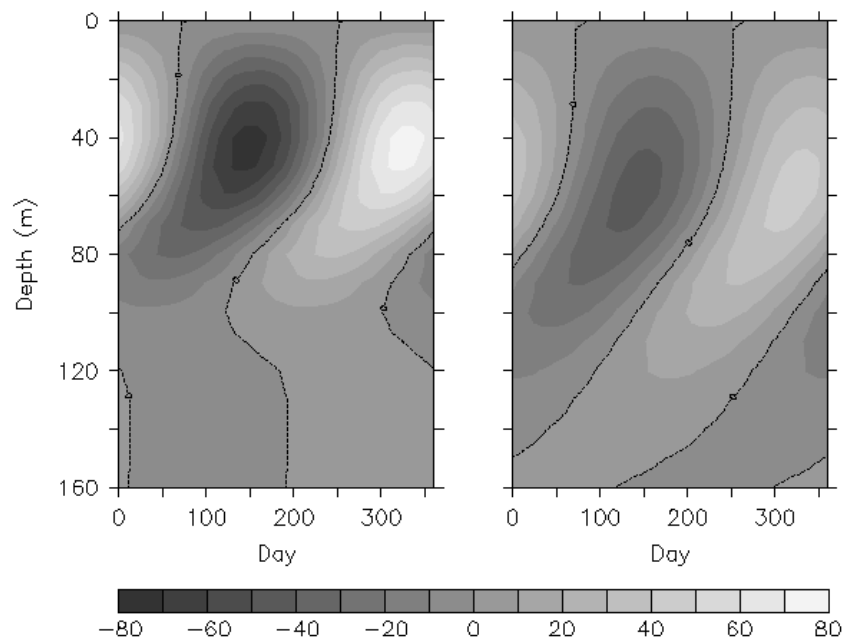

Figure 7 . The 360 -day vertical velocity cycle at $10^{\circ} \mathrm{N}$ (left, $\mathrm{cm} \mathrm{day}^{-1}$ ) and at $20^{\circ} \mathrm{N}$ (right, $\mathrm{cm} \mathrm{day}{ }^{-1}$ ) along the coast. The amplitude of the cycle weakens to the north.

The variation in the 360-day vertical velocity cycle along the coast is predictably different (figure 7). The depth at which the maximum speed occurs increases poleward, while the amplitude of the cycle weakens. Specifically, at $10^{\circ} \mathrm{N}$ the maximum speed of $75 \mathrm{~cm}^{\text {day }}{ }^{-1}$ occurs at $40-\mathrm{m}$ depth, while at $20^{\circ} \mathrm{N}$ the maximum speed of $50 \mathrm{~cm} \mathrm{day}^{-1}$ occurs at 55-m depth.

Both these patterns are consistent with the argument developed in the previous section: the amplitude of the vertical velocity cycle decreases as the beam crosses the depth range of the thermocline.

While the vertical propagation of energy does cause the vertical velocity cycle to vary along the coast, this linear model cannot represent the
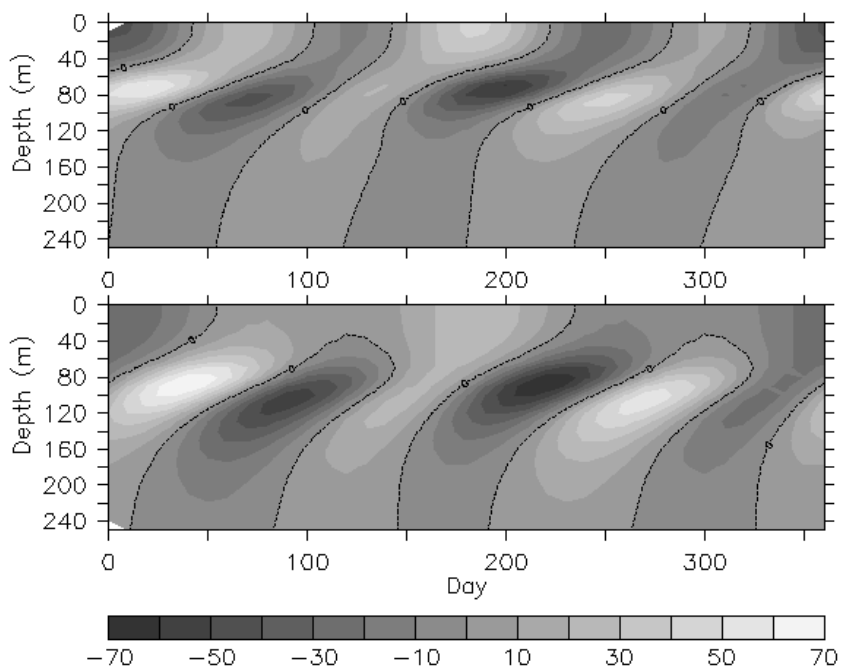

Figure 8. Time series of alongshore current $\left(\mathrm{cm} \mathrm{s}^{-1}\right)$ at $10^{\circ} \mathrm{N}$ (top) and $20^{\circ} \mathrm{N}$ (bottom) along the coast generated by forcing at periods $T=120$ days and 360 days.

processes of entrainment and detrainment of water from the deep ocean into the surface layer. Simulations using a more realistic model would be required to examine possible alongshore variations in entrainment and detrainment.

\subsection{Composite fields}

All simulations presented so far have been forced at a single frequency. It is possible to generate more realistic circulation patterns by summing the velocity fields associated with several forcing periods. Spectral analysis of the current meter data reported by Schott et al (1994) reveals significant energy at 120- and 360-day periods. The strong 120-day cycle is not discussed by either Schott et al (1994) or Reppin et al (1999), but the amplitude of each component is comparable (table 1). Moreover, the phase of the 120-day cycle is approximately zero when the phase of the 360-day cycle is zero.

The time series of alongshore velocity at $10^{\circ} \mathrm{N}$ and $20^{\circ} \mathrm{N}$ generated by forcing at 120 - and 360 -day periods clearly shows the influence of the 120-day cycle (figure 8 ). The current field exhibits three distinct phases. The influence of the 120-day cycle on surface currents weakens to the north, so the model predicts a greater number of surface current reversals at $10^{\circ} \mathrm{N}$ than at $20^{\circ} \mathrm{N}$. As expected, subsurface currents strengthen to the north.

\section{Discussion}

\subsection{Realistic forcing profiles}

Ideally, the forcing vertical profile should closely resemble current profiles observed south of 
Sri Lanka. Our idealised profile $\Psi(z)$ differs from the profiles reported by Schott et al (1994) in many respects. First, our forcing vertical profile is more strongly surface-trapped than the currents observed south of Sri Lanka. Second, undercurrents are not negligible and may extend over significant depths.

Schott et al (1994) also reported that currents south of Sri Lanka exhibit upward phase propagation, indicating downward energy propagation. This is consistent with the hypothesis of remote forcing. Such vertical propagation could be incorporated by introducing a term of the form $e^{i m z}$ into the boundary condition (7). Since the scarcity of data makes it difficult to assign a meaningful value to the vertical wave number $m$, we have chosen to neglect this aspect of the forcing profile.

\subsection{Reflection}

The vertical phase propagation observed in currents south of Sri Lanka raises questions about reflection of energy from the ocean floor. Romea and Allen (1983) demonstrated that, for the ideal case of a smooth, horizontal ocean bottom, energy is reflected at both the ocean surface and floor. In the inviscid case, this process continues indefinitely.

It is not necessary to consider reflection in the simulations reported in this paper, since, for the periods of interest, energy never reaches the ocean floor. This may not be true, however, for coastal
Kelvin waves generated in the northern Bay of Bengal. Such waves, on reaching Sri Lanka, would have already resulted in significant vertical propagation of energy along the Indian east coast. Deep current observations would be required to assess the extent to which energy reflects at the ocean floor.

\subsection{Vertical propagation in current meter data}

One of the basic results of this paper is that vertical propagation of energy associated with coastal Kelvin waves at periods of 30 to 60 days is substantial. A signature of this may be visible in the current meter records reported by Schott et al (1994). Figure 9 shows the amplitude spectra of currents at 150-, 428-, 713- and 1010-m depths between periods of zero and 180 days. The significant attenuation of the amplitude of the 120-day period cycle, relative to the 30- and 60-day cycles, is what would be expected for coastal Kelvin waves remotely forced in the Bay of Bengal.

Similar data are not available to test the model predictions along the Indian west coast. Two long bottom current meter records are, however, available, off Goa on the Indian west coast (A L Paropkari and G Nampoothiri, personal communication 2005). One current meter was located roughly at the shelf break at 200-m depth, while the other was located on the continental slope at 1100-m depth. Consistent with model predictions, a seasonal cycle
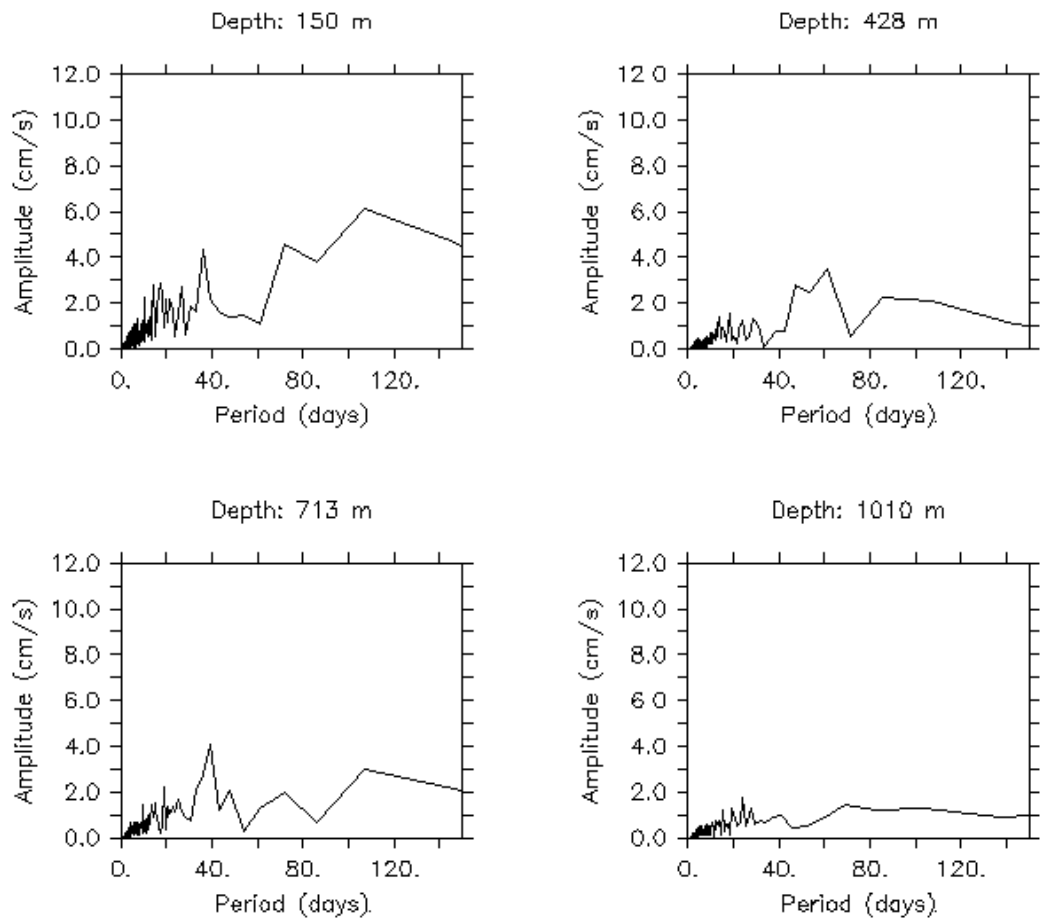

Figure 9. Amplitudes of components at periods $T=0$ to 150 days in zonal currents south of Sri Lanka. Depths are indicated above each graph. At periods greater than 60 days, the attenuation with depth is substantial. 
is evident in the shallow current meter record, but only higher frequencies are seen in the deep current meter record (I Suresh, personal communication 2006).

\subsection{Sensitivity experiments}

The simulations reported above suggest interesting qualitative results about the vertical propagation of coastal Kelvin waves along the Indian west coast. While basic model predictions should hold, sensitivity experiments show that quantitative results can change considerably.

It is apparent that solutions will be sensitive to changes in the forcing vertical profile $\Psi$ as well as the stability profile $N_{b}$. Less obvious is how a spatially variable background density field, caused by an increase in salinity northward along the Indian west coast (Shetye et al 1990, 1991), might influence the vertical propagation of coastal Kelvinwave beams.

Hydrographic observations (Shetye et al 1990, 1991), though not conclusive, also suggest that the undercurrent along the Indian west coast weakens to the north during the summer and winter monsoons, contrary to the predictions presented in section 3.4. This discrepancy is most probably due to friction being larger in reality than is assumed in the model. Vertical mixing tends to dampen higher order modes, resulting in a more 'diffuse' beam and a weakening of the return flow required for baroclinic motion. Sensitivity experiments show that increasing the value of the vertical mixing parameter $M_{v}$ by one order of magnitude causes the undercurrent in the 360-day cycle to disappear completely. This model also neglects horizontal friction, which would also act to dampen higher order modes as the Rossby radius of deformation decreases poleward, inevitably bringing such motion into the coastal boundary layer regime.

A detailed analysis of sensitivity experiments is unlikely to yield further insight into model results owing to the lack of observational data. Rather, this paper is intended to provide the motivation for studying the vertical propagation of coastal Kelvin waves with a general circulation model, allowing the influence of density variations, basin geometry, and mixing processes to be examined.

\section{Acknowledgements}

The authors wish to thank Fritz Schott and Rainer Zantopp for their help in obtaining the current meter data from the WOCE/NODC Data Archive.
An anonymous reviewer and $\mathrm{J} \mathrm{P}$ McCreary are thanked for their critical comments, which helped to improve the manuscript. The detailed comments of McCreary led to several changes from the original version. This work was carried out as part of the Franco-Indian Program for Weather and Climate Research (ProFIRMeC) at the National Institute of Oceanography, Dona Paula, Goa, India. D Nethery was supported by the Ecole Normale Supérieure and the CNRS. D Shankar was supported by the Department of Ocean Development, New Delhi. All images in this paper were produced using Ferret. This is NIO contribution 4243.

\section{References}

McCreary J P 1981 A linear stratified ocean model of the equatorial undercurrent; Phil. Trans. Roy. Soc. London 302 385-413.

McCreary J P 1984 Equatorial Beams; J. Mar. Res. 42 395-430.

McCreary J P, Kundu P K and Molinari R L 1993 A numerical investigation of dynamics, thermodynamics and mixed-layer processes in the Indian Ocean; Prog. Oceanogr. 31 181-244.

Reppin J, Schott F A, Fischer J and Quadfasel D 1999 Equatorial currents and transports in the upper central Indian Ocean: Annual cycle and interannual variability; J. Geophys. Res. 104 15,495-15,514.

Romea R D and Allen J S 1983 On vertically propagating coastal Kelvin waves at low latitudes; J. Phys. Oceanogr. 13 1,241-1,254.

Schott F, Reppin J and Fischer J 1994 Currents and transports of the Monsoon Current south of Sri Lanka; J. Geophys. Res. $9925,127-25,141$.

Shankar D, McCreary J P, Han W and Shetye S R 1996 Dynamics of the East India Coastal Current 1. Analytic solutions forced by interior Ekman pumping and local alongshore winds; J. Geophys. Res. 101 13,975-13,991.

Shankar D and Shetye S R 1997 On the dynamics of the Lakshadweep high and low in the southeastern Arabian Sea; J. Geophys. Res. 102 12,551-12,562.

Shankar D, Vinayachandran P N and Unnikrishnan A S 2002 The monsoon currents in the north Indian Ocean; Prog. Oceanogr. 52 63-120.

Shenoi S S C, Shankar D, Michael G S, Kurian J, Varma K K, Ramesh Kumar M R, Almeida A M, Unnikrishnan A S, Fernandes W, Barreto N, Gnanaseelan C, Mathew R, Praju K V and Mahale V 2005 Hydrography and water masses in the southeastern Arabian Sea during March-June 2003; J. Earth Syst. Sci. 114 475-491.

Shetye S R, Gouveia A D, Shenoi S S C, Sundar D, Michael G S, Almeida A M and Santanam K 1990 Hydrography and circulation off the west coast of India during the southwest monsoon 1987; J. Mar. Res. 48 359-378.

Shetye S R, Gouveia A D, Shenoi S S C, Michael G S, Sundar D, Almeida A M and Santanam K 1991 The coastal current off western India during the northeast monsoon; Deep-Sea Res. 38 1517-1529. 